\title{
Surveillance Camera Based Fall Detection System Using Long Short Term Memoryfor Elderly People
}

\author{
G.Anitha ${ }^{1}$, S.BaghavathiPriya ${ }^{2}$ \\ \{anitha.g@rajalakshmi.edu.in ${ }^{1}$, baghavathipriya.s@ rajalakshmi.edu.in ${ }^{2}$ \} \\ Department of IT, Rajalakshmi Engineering College, Chennai ${ }^{1}$, Department of CSE, Rajalakshmi \\ Engineering College, Chennai $^{2}$
}

\begin{abstract}
Event detection in videos is becoming an emerging area of research now a day. Monitoring of people activities using a surveillance camera is an essential one in a recent lifestyle for safety and security. The surveillance cameras are used in a wide variety of places such as in public places, Hospitals, Schools, and Homes for the beneficiaries of common people, patients, children and the elderly. In case of any emergency or abnormal events, immediate notification should be given to the respective people. The abnormal events are recognized from the videos using deep architectures. The goal of event detection in videos is to detect simple and complex actions in real-time data. This has a lot of attention in real-time ambient assisted living environments especially for elder people who live alone in the home. In this paper, a deep architecture of long short term memory recurrent network is proposed to detect fall actions in video inputs..
\end{abstract}

Keywords: RNN, LSTM, CNN, Action Recognition and Fall.

\section{Introduction}

Fall detection has become the most interesting research area due to the most attractive and efficient features of the deep learning algorithms in computer vision domain. In the last few decades more number of research works is evolved in the field of Ambient Assisted Living (AAL) for elderly people. AAL and smart home automation is achieved with various kinds of sensors and monitoring devices to enhance the life style of the elderly people. In recent decades, the life time of people is increasing drastically due to the recent advancements and growth of technology in medicine field. This leads to the population growth in old age people.

The number of people and proportions of older persons are increasing day by day all over the world. By 2050, one in six people in the world will be over age 65 stated by world population prospects: The 2019 revision [1]. The old age people wish to live independently and peacefully in their last days in home. Due to aging they may experience health issues like sight problems, unbalancing while walking, dementia etc. It is mandatory to have health care assistant and being monitored around 24 hours and it is possible only when they are in the clinical environment. But the older persons are willing to have ageing at home. They hesitate to be in the hospital like environment.

In this research work a novel deep learning algorithm is proposed to detect human fall in indoor environment automatically using computer vision techniques. The paper is further organized into related work, methodology, results and discussion and conclusion. 


\section{Related work}

'To overcome this issue researchers have developed many monitoring devices to support aging at home. The monitoring devices are further classified into three broad categories acoustic and ambient sensors based method, wearable sensors based method, computer vision based method [2].In acoustic ambient method the sounds of the surrounding and frequencies are recorded to detect the fall. In this method various sensors like floor vibration sensor, location sensor, RFID, temperature sensor and infrared sensors are embedded in to the environment to detect the actions and context of the elderly [3]. In wearable sensors based method various kinds of sensors like accelerometers [2-table] and gyroscopes are used to detect the fall event. In computer vision based methods surveillance cameras are used to monitor the people daily activities and if any fall events occur that can be detected based on their postures or from other parameters using well defined algorithms and alarm is sent to caretakers immediately.

Among the all fall detection methods computer vision based methods are giving better accuracy compared to other traditional methods. They produced less false alarm rate and the results can be further improved by the efficient deep learning algorithms. Deep learning supports real time applications and large volume of data and gives better accuracy than other machine learning algorithms. ArifMahamood et.al extracted spatio temporal features from images and classified using ada boost algorithm to detect fall [4]. Manuel Martinez proposed a bed aligned map method to recognize human activities in bed [5]. Many researches have proposed many feature extraction and classifiers to detect human fall automatically like HOG[8], fuzzy rules[9], SVM[7], GMM [6], DAG [10].

In [11] Guo-Jun Qi extracted position, angle, offset, velocity, pairwise joint distances of the persons from image and proposed differential recurrent neural network deep algorithm to find the change of information between successive frames for the classification. MirtoMusci[12] detected fall using wearable devices and artificial intelligence techniques.Ruizaho et al. combines RNN with CNN and finally used SVM classifier to classify the classes. Ahmed Naitaicha compared CNN, LSTM and ConvLASTM on wearable devices data for detection [13].Adrian Nunez Macros implemented CNN on publically available fall detection datasets URFD, Multicam, FDD and achieved 94\% results[14].Deepika Sign et al classified fall events on sensor data using LSTM and RNN deep learning algorithms and it outperforms traditional algorithms NB, HMM and CRF[15]. Chi Chuntian proposed a novel method that extracts channel state information from WiFi signals and trained with RNN model. Then it is improved with RSR-RNN model[16].

\section{Methodology}

The automatic fall detection system captures input frames from the video surveillance camera and extracts the important features. Then it converts the data to CSV file format to train the RNN model with the pre-processed data in the training phase. In testing phase a new input data is processed by the trained model and the events are classified accordingly. Fig.1 shows the basic model of the automatic fall detection system using the deep learning algorithm RNN. 


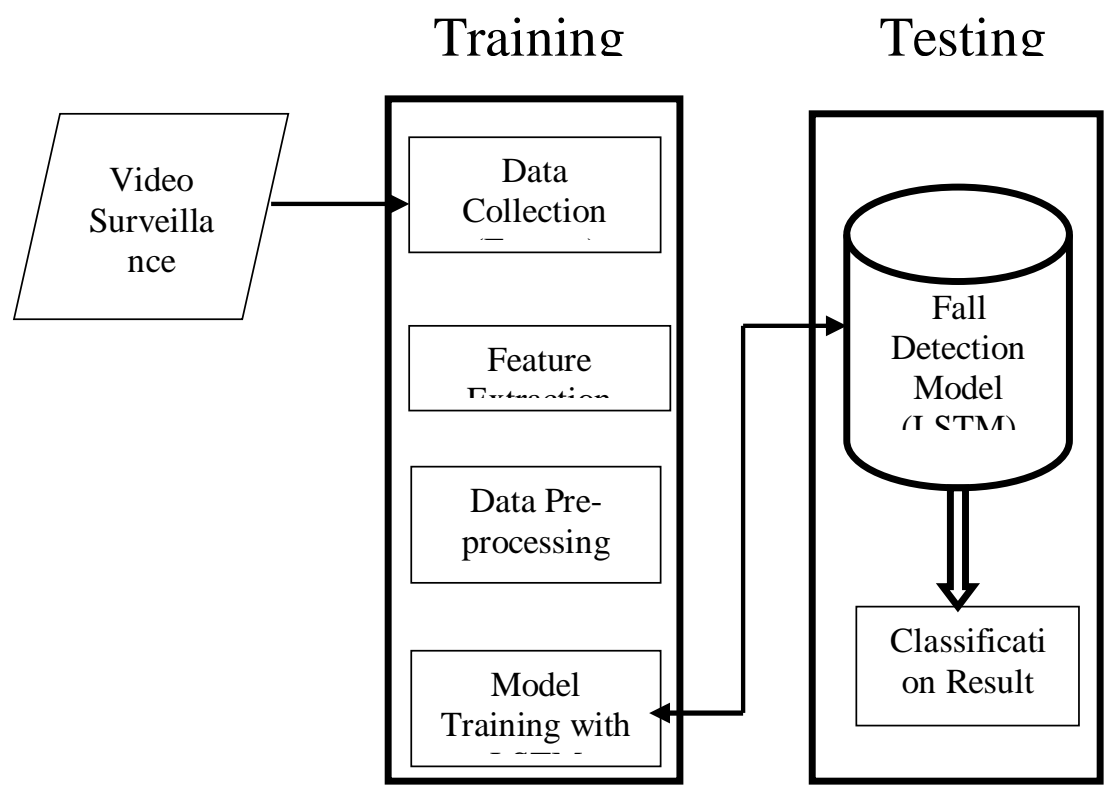

\section{RNN}

Fig.1 Automatic Fall Detection System

Recurrent Neural Network is a well defined deep network for time series data. RNN structure includes an input layer multiple hidden layers and an output layer. This forwards input $\mathrm{x}$ to a internal hidden layer $\mathrm{h}$ at time step $\mathrm{t}$. The deep network passes the hidden state $h_{t}$ along with the next input $x_{t+1}$ to the neuron at every time step. The number of hidden layers is determined by trial and error methods in training phase. Each hidden layer is having its own weights and biases. RNN provides same weights and biases to all the layers to reduce the complexity so that all the hidden layers have same weights and biases in a single recurrent layer. Fig. 2 shows a simple RNN structure.

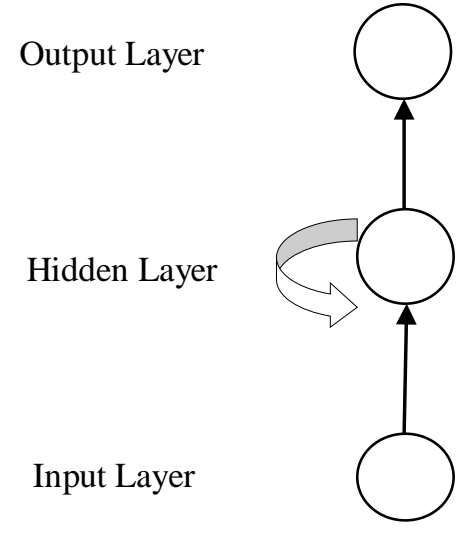

Fig.2 A simple RNN structure

Formula (1) shows the calculation of the current state where $h_{t}$ and $h_{t-1}$ are the current and previous states and $\mathrm{X}_{\mathrm{t}}$ is the input state. Formula (2) depicts activation function $\mathrm{f}()$ where 
$\mathrm{W}_{\mathrm{hh}}$ is weight at recurrent neuron and $\mathrm{W}_{\mathrm{xh}}$ is weight at input neuron. Formula (3) states the calculation of output $Y_{t}$ where $w_{h y}$ is weight at output layer.

$$
\begin{aligned}
& h_{t}=f\left(h_{t-1}, X_{t}\right) \\
& h_{t}=\tanh \left(W_{h h} h_{t-1}+W_{x h} X_{t}\right. \\
& Y_{t}=W_{h y} h_{t}(3)
\end{aligned}
$$

LSTM

RecurrentNeural Network algorithm is the best choice to analyze sequential input data. Video inputs have information in many frames. All of them need to be analyzed to understand the context of the event. Longer sequences cannot be handled in RNN as it forgets previous input sequence. To diminish this gradient descent problem, Long Short Term Memory has been designed to analyze the longer sequences and preserve contextual information of input sequence. Back propagation technique can be used to overcome the problem of vanish. Its architecture with input output and forget gates help to identify long term patterns in sequential input.In LSTM the input output and control gates are represented as $\mathrm{x}_{\mathrm{t}}, \mathrm{c}_{\mathrm{t}}, \mathrm{O}_{\mathrm{t}}$ with hidden state $\mathrm{h}_{\mathrm{t}}$ at time $\mathrm{t}$. At each time step the LSTM neuron depends on the values of input gate, cell state and hidden states to produce output. Each LSTM unit's functionality is depicted in equations (3)-(8). Using this values LSTM unit memorize long term input sequence.

$$
\begin{aligned}
& i_{t}=\sigma_{g}\left(w_{x i} x_{t}+W_{h i} h_{t-1}+b_{i}\right) \\
& f_{t}=\sigma_{g}\left(W_{x f} x_{t}+W_{h f} h_{t-1}+b_{f}\right) \\
& o_{t}=\sigma_{g}\left(W_{x o} x_{t}+W_{h o} h_{t-1}+b_{o}\right) \\
& g_{t}=\tanh \left(W_{x g} x_{t}+W_{h g} h_{t-1}+b_{g}\right) \\
& c_{t}=f_{t} \odot c_{t-1}+i_{t} \odot g_{t} \\
& h_{t}=o_{t} \odot \sigma_{h}\left(c_{t}\right)
\end{aligned}
$$

The human actions are recognized and classified using this LSTM model and achieved better accuracy.

\section{Results and Discussions}

The proposed model is evaluated with Gaussian Naïve Bayes classifier and proposed method. Table 1 shows the performance of our model with different number hidden layers. Figure 2 shows the live capture of fall event. Table 2 and 3 shows the precision, recall, f1 score and support values of Gaussian and LSTM methods. The three class labels are fall, non fall and about to fall. Table 4 shows the accuracy values of both the algorithms and LSTM achieved $85.79 \%$. 


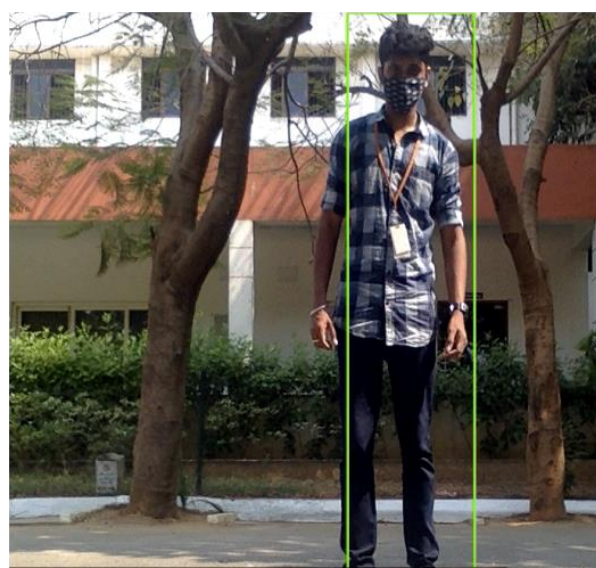

a

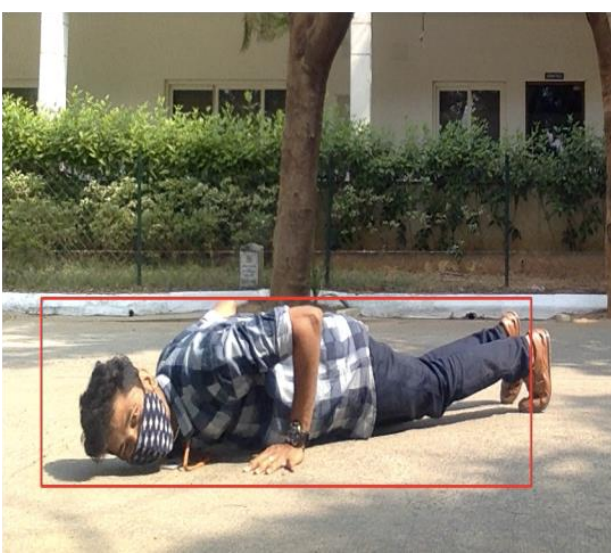

b

Figure 2. Fall Event Detection a. Non-Fall (Person Detection) b. Fall

Table 2: Gaussian Naïve Bayes

\begin{tabular}{|l|l|l|l|l|}
\hline Class & Precision & Recall & F1-Score & Support \\
\hline 0 & 0.42 & 0.65 & 0.51 & 438 \\
\hline 1 & 0.89 & 0.87 & 0.88 & 587 \\
\hline 2 & 0.95 & 0.83 & 0.88 & 1861 \\
\hline
\end{tabular}

Table 3: LSTM

\begin{tabular}{|l|l|l|l|l|}
\hline Class & Precision & Recall & F1-Score & Support \\
\hline 0 & 0.45 & 0.38 & 0.41 & 405 \\
\hline 1 & 0.82 & 0.95 & 0.88 & 591 \\
\hline 2 & 0.93 & 0.91 & 0.92 & 1890 \\
\hline
\end{tabular}

Table 4: Accuracy

\begin{tabular}{|l|l|}
\hline Method & Accuracy \\
\hline Gaussian Naïve Base & $82 \%$ \\
\hline LSTM & $\mathbf{8 5 . 7 9 \%}$ \\
\hline
\end{tabular}

\section{Conclusion}

A deep architecture of long short term memory recurrent network is proposed to detect fall actions in video inputs. The method achieved good results than machine learning algorithms. The results can be improved with more number of input video samples. In future work, Attention based models can be used to improve the model performance.

Word document can be used as a template for papers to be published in EAI Core Proceedings. Follow the text for further instructions on text formating, tables, figures, citations and references. 


\section{References}

[1] https://www.un.org/en/sections/issues-depth/ageing/

[2] SreeMadhubala J, Umamakeshwari A, JenitaAmali Rani B, "A Survey on technical approaches in fall detection”, National Journal of Physiology, Pharmacy and Pharmocology, 2015

[3] SreeMadhubala J, Umamakeshwari A, JenitaAmali Rani B, "A Survey on technical approaches in fall detection", National Journal of Physiology, Pharmacy and Pharmocology, 2015

[4] Syed Farooq Ali, Reamsha Khan, Arif Mahmood, Malik Tahir Hassan and Moongu Jeon, "Using Temporal Covarience of Motion and Geometric Features via Boosting for Human Fall Detection", sensors,2018,doi:10.3390/s18061918.

[5] Manuel Martinez, Lukas Rybok and Rainer Stiefelhagen, "Action Recognition in Bed using BAMs for assisted living and elderly care", $14^{\text {th }}$ IAPR International Conference on Machine Vision Applications (MVA), May 18-22, 2015, Miraikan, Tokyo, Japan.

[6] Hamid Rajabi, ManoochehrNahvi, "An Intelligent Video Surveillance System for Fall and Anesthesia Detection for Elderly and Patients", $2^{\text {nd }}$ International Conference on Pattern Recognition and Image Analysis (IPRIA 2015) March 11-12, 2015.

[7] Omar Aziz, JochenKlenk, Lars Schwickert, Lorenzo Chiari, Clemens Becker, Edward J.Park, Greg Mori and Stephen N.Robinovitch, "Validation of accuracy of SVM-based fall detection system using real-world fall and non-fall datasets", https://doi.org/10.1371/journal.pone.0180318.

[8] Mai Nadi, Nashwa EI-Bendary, Hamdi Mahmoud and Aboul Ella Hassanien, "Fall Detection System of elderly people based on integral image and histogram of oriented gradient feature", International Conference on Hybrid Intelligence Systems(HIS), IEEE,2014.

[9] KhosroRezaee, JavadHaddadnia, Ahmad Delbari, "Intelligent Detection of the Falls in the Elderly using Fuzzy Inference System and Video based Motion Estimated Method", $8^{\text {th }}$ Iranian Conference on Machine Vision and Image Processing (MVIP), IEEE, 2018.

[10] Miao Yu, Adel Rhuma, Syed Mohsen Naqvi, “A posture Recognition Based Fall Detection System for Monitoring an Elderly Person in a Smart Home Environment", IEEE Transactions on Information Technology in Bio Medicine, Vol.16, No.6, November 2012.

[11] VivekVeeriah, Naifan Zhuang, Guo-Jun Qi, "Differential Recurrent Neural Networks for Action Recognition", IEEE Explore, ICCV.

[12] MirtoMusci, Daniele De Martini, Nicola Blago, TullioFacchinetti and Macro Piastra, "Online Fall Detection using Recurrent Neural Network", arXiv:1804.0497 [cs.CY] 13 Apr 2018.

[13] Ahmed NaitAicha, Gwenn Englebienne, Kimberley S. van Schooten, MirjamPijnapples and Ben Krose, "Deep Learning to Predict Falls in older Adults Based on Daily -Life Trunk Accelerometry", Sensors, 2018, 18, 1654, doi:10.3390/s18051654.

[14] Adrian Nunez-Macros, GorkaAzkune, and Ignacio Arganda-Carrearas, " Vision - Based Fall Detection with Convolutional Neural Networks", Hindawi, Wireless Communications and Mobile Computing, Vol.2017, Article ID 9474806.

[15] Deepika Singh, ErincMerdivan, IsminiPsychoula, Johannes Kropf, StenHanke, Matthiedu Geist and Andreas Holzinger, "Human Activity Recognition Using Recurrent Neural Network", IFIP International Federation for Information Processing, Springer, 2017, Doi: 10.1007/978-3-31966808-6_18.

[16] Chi Chuntian, "Fall Detection for Elders in Indoor Environment using WiFi Signals", Thesis, Camford Royal School, Beijing, China, September 2017. 